\title{
ANÁLISIS CUANTITATIVOS APLICADOS A LA POESÍA: LA LÍRICA DUBITADA SACRA DE DAMIÁN CORNEJO
}

\author{
Zoraida Sánchez Mateos \\ Universidad de Valladolid ${ }^{1}$
}

\section{Resumen}

Los análisis cuantitativos permiten delimitar y contrastar de manera objetiva las características estilísticas de corpus textuales. A través de este tipo de estudios, se intentarán definir rasgos morfosintácticos y léxicos que permitan diferenciar los poemas sacros indubitados de Damián Cornejo y de León Marchante. El objetivo será obtener una serie de parámetros estadísticos que ayuden a determinar la autoría de las poesías religiosas que se atribuyen a ambos.

Palabras clave: Poesía sacra, Damián Cornejo, análisis cuantitativos, determinación de autoría.

\section{QUANTITATIVE ANALYSIS APPLIED TO POETRY: THE DOUBTED SACRED POEMS OF DAMIAN CORNEJO}

\begin{abstract}
Quantitative analysis allows to delimit and contrast objectively the stylistic characteristics of textual corpus. Through this type of studies, we will try to define morphosyntactic and lexical features that allow us to differentiate the undoubted sacred poems of Damian Cornejo and Leon Marchante. The objective will be to obtain a series of statistical parameters that help determine the authorship of the religious poems attributed to both of them.

Keywords: Sacred poetry, Damian Cornejo, quantitative analysis, determination of authorship.
\end{abstract}

1 Esta investigación es el resultado de los trabajos realizados en el seno del proyecto de investigación «Ovidio versus Petrarca: nuevos textos de la poesía erótica española del Siglo de Oro (plataforma digital y edición)», Ref. FFI2015-68229-P.

Fecha de recepción: 24 de enero de 2019

Fecha de aceptación: 13 de mayo de 2019 


\section{INTRODUCGIÓN}

En los códigos de producción del Bajo Barroco no primaba la voluntad de plasmar el estilo personal del autor, sino que se buscaba crear obras acordes a los gustos y a la demanda del público². Esto provocó que algunas de las poesías que poseían temas y rasgos semejantes y que se transmitían a través de manuscritos se atribuyeran a varios ingenios. El interés de fray Damián Cornejo y de León Marchante por la lírica festiva, su tendencia al prosaísmo y, sobre todo, el hecho de que sus versos pudieron haberse difundido en los mismos círculos y de manera simultánea ${ }^{3}$ hizo que ochenta y siete poemas (veintinueve sacros y cincuenta y ocho profanos) se adjudicaran a ambos escritores.

La fama de poeta que poseía el dramaturgo manchego ${ }^{4}$, frente a un escritor ocasional como Cornejo, propició que gran parte de los textos dubitados con el cronista seráfico se publicaran en el Siglo de las Luces bajo su nombre ${ }^{5}$. Esto, unido al escaso cultivo que hizo fray Damián de la lírica religiosa, ha provocado que se llegara a poner en duda incluso la autoría de las poesías de temática divina que únicamente se atribuyen a él (Carreira, 2008) ${ }^{6}$. Con el propósito de intentar aportar datos objetivos y cuantificables que ayuden a discernir entre las obras sacras de ambos autores, algunas de ellas serán sometidas a una serie de análisis cuantitativos?

2 La poesía del Bajo Barroco es un fiel testimonio de los importantes cambios culturales y sociales que se produjeron en España en dicho periodo. Sus singulares características permiten trazar un puente entre el Barroco y el Neoclasicismo, el cual ha sido estudiado en profundidad por Mata Induráin y Bègue (2018).

3 Existen múltiples puntos de confluencia entre las vidas de Marchante y de Cornejo: «nacidos en torno a 1630, estudiaron y vivieron bastante tiempo en Alcalá, uno era cura, el otro fraile, ambos defensores de la Inmaculada Concepción y tentados de las musas; no sabemos si fueron amigos o rivales, pero es imposible que no se conocieran» (Carreira, 2008: 46).

${ }^{4}$ El maestro León fue el poeta de la segunda mitad del seiscientos que más publicó en vida. Desde 1658 hasta un par de años antes de su muerte (1680), sus versos sacros fueron llevados a la imprenta en diecinueve ocasiones. Además, el dramaturgo manchego imprimió las composiciones presentadas para la justa de 1657 (realizada en Alcalá de Henares), un poema de temática profana, una carta a un amigo suyo y la relación de un festejo taurino (Bègue, 2010).

5 Las poesías que conforman las Obras poéticas póstumas de Marchante, principalmente de contenido sacro, fueron recogidas por un aficionado anónimo a partir de múltiples fuentes de dudosa fiabilidad. Las setenta y siete poesías dubitadas con Cornejo que estas contienen se distribuyen de la siguiente manera en los tomos que las conforman. En el primer volumen (1722), se hallan veinticuatro, en el segundo (1733), catorce y, en el tercero (1733), treinta y nueve.

${ }^{6}$ Aunque el número de poemas que se atribuyen a fray Damián es abundante (unos doscientos), solo treinta y ocho son de temática religiosa. De estos, nueve se pueden considerar de autoría segura.

7 El empleo de análisis estadísticos en corpus poéticos que plantean dudas relacionadas con la autoría ha sido puesta en práctica por múltiples investigadores. Pueden citarse, por su relevancia en dicho campo, los trabajos de Burrows y Love (1999), Craig y Kinney (2009) y Craig (2012). 
En esta investigación, se ha transcrito y normalizado un corpus de dieciocho poemas religiosos indubitados de fray Damián ${ }^{8}$ y del maestro León (nueve de cada poeta) y dieciséis que se atribuyen a ambos ${ }^{9}$. Todos ellos poseen más de cien palabras ${ }^{10}$ para garantizar que los programas utilizados $(R$, SPSS, Treetagger, ContaWords y $L I W C$ ) puedan extraer datos relevantes, tanto individuales como de conjunto, sobre el uso que se hace en ellos de la gramática y del vocabulario $^{11}$. En la codificación de las poesías seleccionadas ${ }^{12}$, se ha utilizado la inicial del apellido de Cornejo y de León («G»y «L») y las etiquetas «si» $\mathrm{y}$ «D_s» con el propósito de indicar si se trata de composiciones indubitadas o dubitadas.

\section{ANÁlisis MORFOSINTÁCTICO}

El estudio cuantitativo del uso de la morfosintaxis permite distinguir y clasificar con un alto grado de fiabilidad textos, breves y extensos, de diferentes autores. Gracias a la herramienta Treetagger, se han podido obtener los porcentajes de algunas de las principales categorías gramaticales que aparecen en la poesía sacra (dubitada e indubitada) de fray Damián y del maestro León. A partir de tales datos, con el programa $R$, se han generado un análisis de multivariantes (con catorce variables ${ }^{13}$ ). Este representa gráficamente, a través de las flechas del biplot ${ }^{14}$, las diferencias y las similitudes morfosintácticas que existen entre las diversas poesías analizadas:

8 Se han considerado indubitados aquellos poemas que se atribuyen exclusivamente a Cornejo y que aparecen en más de cuatro manuscritos integri que recopilan su obra poética.

9 Las poesías sacras dubitadas que han sido analizadas en esta investigación fueron publicadas en las Obras Póstumas de Marchante (1722 y 1733) y aparecen en el manuscrito 2245 de la BNE, uno de los testimonios más completos y fiables que intenta recopilar la lírica del cronista seráfico. Tales composiciones fueron editadas a nombre de Cornejo por Klaus Pörtl (Cornejo, 1978).

${ }^{10}$ Las investigaciones de Eder (2015 y 2017) demuestran que cuanto mayor sea la longitud de los textos más posibilidades hay de evitar errores en su clasificación. No obstante, es fundamental que en estos esté presente la huella estilística de sus autores, pues solo así las herramientas de análisis cuantitativos podrán discernir con claridad entre las obras de diversos escritores.

11 La extracción automática de datos gramaticales y léxicos puede ayudar a determinar no solo las características de la poesía de un poeta, también permite establecer relaciones (conjuntivas y disyuntivas) con los versos de otros autores. Investigaciones como las de Smarandache (2007), Opara (2015) y Rezaei y Kashanian (2017) son un ejemplo de ello.

12 Se han elaborado dos tablas (ver Anexo: Tablas 5 y 6) donde se especifican los títulos de los poemas analizados, se concreta su localización, se indica su extensión, el tipo de métrica y la codificación empleada para identificarlos.

13 Se han elegido las siguientes variables de análisis: adjetivos (ADJ), adverbios (ADV), artículos (ART), nombres comunes (NC), preposiciones (PREP), cuantificadores (QU), demostrativos (DM), posesivos (PPO), pronombres personales (PPX), clíticos (PPC), marcas de negación (NEG), conjunciones copulativas (CC), conjunciones subordinantes (CSUBX) y conjunción «que» (CQUE).

${ }_{14}$ La representación mediante biplots permite mostrar cómo la combinación de diversas variables (categorías morfosintácticas) en un mismo gráfico establece semejanzas y diferencias 


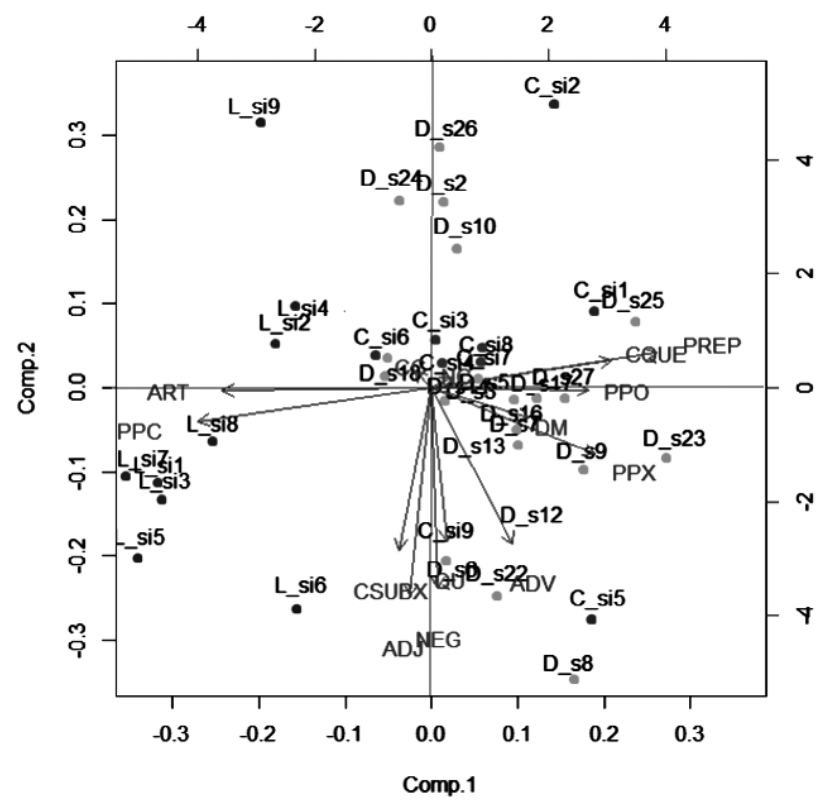

Gráfico 1. Características morfosintácticas de los poemas sacros indubitados de Cornejo y de Marchante y de los dubitados entre ambos.

Aunque ninguno de los tres corpus analizados (L_s, G_s y D_s) constituye un grupo de poesías cohesionado ${ }^{15}$, los datos reflejados en el biplot manifiestan características que los singularizan. Los poemas situados en los cuadrantes izquierdos pertenecen a Marchante (L_s) y presentan un empleo elevado de clíticos «PPC»y artículos «ART», mientras que los que se hallan en los de la derecha son los dubitados (D_s) y los indubitados del cronista seráfico (C_s) y tienden a utilizar más los posesivos y las preposiciones. Resulta significativo señalar que el grueso de las composiciones que integran estos dos últimos grupos se hallan en la parte central del gráfico y que los textos que ocupan los extremos (superior e inferior) parecen distanciarse del resto de su conjunto por sus diferencias de extensión ${ }^{16}$. A pesar de ello,

entre creaciones de un mismo autor o de varios. Cuanto mayor sea el número de estas, más precisos serán los datos resultantes. Sánchez (2018) utiliza este tipo de análisis para delimitar los elementos gramaticales que singularizan la forma de escritura (en prosa y en verso) de varios poetas del Bajo Barroco.

15 Si se extraen del análisis de multivariantes los poemas dubitados, se puede observar cómo se acrecientan las diferencias morfosintácticas entre los indubitados de Marchante y los de Cornejo y cómo se aglutinan más en el biplot (por su semejanza en el uso de la gramática) las poesías de un mismo autor (ver Anexo: Gráfico 4).

${ }_{16}$ Los poemas que ocupan el cuadrante izquierdo superior presentan (en su mayoría) una extensión más elevada que los situados en la parte inferior. Tal dato podría poner de 
todas las poesías disputadas entre ambos autores se hallan más próximas a las creaciones de autoría segura de fray Damián y, por tanto, poseen una mayor similitud morfosintáctica con estas que con las de León.

Para corroborar estos datos, se ha recurrido a otras pruebas de clasificación de individuos o entidades que también se basan en el estudio de multivariantes ${ }^{17}$. Por un lado, se ha realizado (a través del programa SPSS) un análisis clúster con el objetivo de determinar el número de grupos que conforman los poemas seleccionados y para comprobar en cuáles de estos se integran cada uno de ellos $^{18}$. Por otro, se ha determinado mediante un análisis de discriminantes las categorías gramaticales que más influyen en su agrupación. A continuación, se muestran dos tablas con los porcentajes obtenidos:

Tabla 1. Análisis clúster y análisis de discriminantes realizado a partir de variables morfosintácticas

\begin{tabular}{|l|c|c|}
\hline \multicolumn{3}{|c|}{ Análisis Clúster } \\
\hline & 1 & 2 \\
\hline NC & 18,37 & 18,37 \\
\hline PREP & 1,36 & 11,72 \\
\hline PPG & 10,66 & 2,15 \\
\hline PPO & 1,32 & 2,98 \\
\hline ADV & 3,69 & 4,57 \\
\hline ADJ & 7,30 & 6,57 \\
\hline QU & 5,37 & 4,81 \\
\hline CG & 3,12 & 3,21 \\
\hline CSUBX & 2,22 & 2,12 \\
\hline ART & 9,41 & 6,79 \\
\hline PPX & 0,91 & 2,16 \\
\hline CQUE & 1,56 & 3,56 \\
\hline DM & 0,54 & 0,71 \\
\hline NEG & 1,52 & 1,62 \\
\hline
\end{tabular}

\begin{tabular}{|c|c|c|c|}
\hline \multicolumn{4}{|c|}{ Análisis de discriminantes } \\
\hline \multicolumn{2}{|c|}{ Cluster Number of Case } & Mean & $\begin{array}{c}\text { Std. } \\
\text { Deviation }\end{array}$ \\
\hline \multirow{5}{*}{1 León } & PPC & 10,6556 & 2,66651 \\
\hline & PO & 1,3222 & 1,05211 \\
\hline & PREP & 1,3556 & 1,12373 \\
\hline & ART & 9,4111 & 2,72096 \\
\hline & CQUE & 1,5556 & 2,37913 \\
\hline \multirow{5}{*}{$\begin{array}{r}2 \text { Cornejo y } \\
\text { dubitados }\end{array}$} & PPC & 2,1510 & 1,14977 \\
\hline & PO & 2,9845 & 2,05850 \\
\hline & PREP & 11,7241 & 2,02752 \\
\hline & ART & 6,7923 & 2,36483 \\
\hline & CQUE & 3,5567 & 1,42151 \\
\hline
\end{tabular}

manifiesto que los porcentajes morfosintácticos pueden verse influenciados por la longitud de los textos.

17 Se ha recurrido al empleo de análisis clúster y de análisis discriminante. El primero permite agrupar entidades que comparten similitudes en las variables introducidas, mientras que el segundo (a partir de los conjuntos creados en el clúster) determina cuáles son las variables que mejor diferencian los grupos establecidos.

${ }_{18}$ La agrupación de cada uno de los poemas analizados en el análisis clúster puede consultarse en la Tabla 7 del Anexo. 
Las treinta y ocho poesías estudiadas han constituido dos grupos en el análisis clúster. El primero destaca por el uso elevado de clíticos y artículos y está constituido por los poemas indubitados de Marchante. El resto de las composiciones (indubitadas del cronista seráfico y dubitadas) conforman el segundo grupo, donde se aprecia un abundante empleo de preposiciones, posesivos y de subordinadas introducidas por la conjunción «que» ${ }^{19}$. El análisis de discriminantes muestra que las variables que permiten diferenciar mejor los dos bloques son «PREP», «PPO», «PPC» corpus analizados, se ha podido corroborar los datos aportados en el clúster y en el biplot (ver Anexo: Tabla 7).

Con el propósito de continuar probando las semejanzas y diferencias estilísticas que existen entre las poesías sacras indubitadas de fray Damián y de León y las que se atribuyen a ambos, se han analizado con el programa ContaWords las combinaciones gramaticales «bigramas» más recurrentes de nombres, verbos y adjetivos que aparecen en todas ellas:

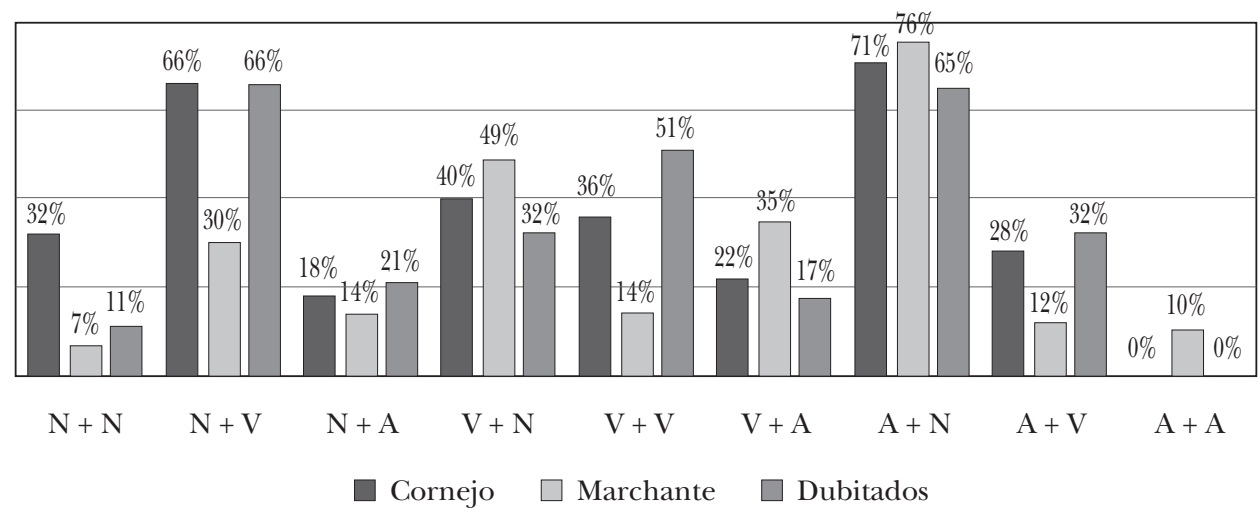

Gráfico 2. Frecuencia de bigramas.

El bigrama más frecuente en las poesías indubitadas sacras de Cornejo y de Marchante es la combinación de un adjetivo más un nombre $(\mathrm{A}+\mathrm{N})$ y el menos utilizado es el de dos adjetivos seguidos $(\mathrm{A}+\mathrm{A})$. En estas, el cronista seráfico emplea con mucha más frecuencia la estructura de un sustantivo más un nombre o un verbo y el dramaturgo manchego explota más la de verbos

19 Todos estos datos han sido contrastados con los aportados en el análisis de la poesía profana indubitada de Cornejo y de Marchante y se ha podido concluir que la temática influye en el uso que estos autores hacen de la gramática. En las composiciones humanas, el maestro León emplea abundantemente los sustantivos comunes y los artículos y fray Damián utiliza muchos más adjetivos, adverbios y cuantificadores. 
con adjetivos y nombres ${ }^{20}$. Con excepción de la combinación $\mathrm{N}+\mathrm{N}$, los porcentajes de los bigramas que se utilizan en los poemas religiosos dubitados entre ambos se asemejan más a los obtenidos en las poesías de fray Damián.

\section{AnÁlisis léXico-SEmÁntico}

Se ha realizado el análisis del léxico y de la semántica de la lírica sacra indubitada y dubitada de Cornejo y Marchante para completar los datos estilísticos aportados en el estudio morfosintáctico y comprobar si existen diferencias o similitudes significativas en el vocabulario que se emplean en tales corpus. A partir del programa $L I W C$, se ha indagado en el tipo de emociones (positivas y negativas) ${ }^{21}$ que predomina en estos, en su subjetivi$\mathrm{dad}$, en su contenido social y en su riqueza léxica. Los resultados obtenidos se muestran en la siguiente tabla:

Tabla 2. Análisis léxico-semántico

\begin{tabular}{|l|c|c|c|c|c|}
\hline \multicolumn{2}{|c|}{ Self-Reference } & Social Words & $\begin{array}{c}\text { Positive } \\
\text { emotions }\end{array}$ & $\begin{array}{c}\text { Negative } \\
\text { emotions }\end{array}$ & $\begin{array}{c}\text { Ratio } \\
\text { Type-Token }^{22}\end{array}$ \\
\hline C_si & 0,39 & 0,41 & 0,17 & 0,58 & 38,1 \\
\hline L_si & 0,31 & 0,74 & 0,48 & 0,83 & 34,9 \\
\hline D_s & 0,44 & 0,53 & 0,49 & 0,53 & 38,2 \\
\hline
\end{tabular}

Puede señalarse que tanto la lírica sacra indubitada de Cornejo como los poemas dubitados dan más relevancia a las autorreferencias que los de Marchante y que, en las poesías disputadas entre ambos, estas poseen un porcentaje mayor. Sucede al contrario con las palabras sociales, ya que las composiciones del dramaturgo manchego manifiestan una mayor voluntad de conectar con el receptor ${ }^{23}$ que el resto de corpus analizados. Respecto a las emociones, es importante mencionar que en ellos predominan los sentimientos negativos, los cuales también son más abundantes en las poesías

20 Tales resultados coinciden en su mayoría con los obtenidos en los versos indubitados humanos de ambos autores, pues estos únicamente presentan diferencias en el uso de los bigramas $\mathrm{N}+\mathrm{V}$ y $\mathrm{A}+\mathrm{N}$.

21 Una compleja muestra del gran interés que supone para los estudios literarios la incorporación de análisis computacional de emociones la aportó Silgen (2016), en cuyo blog expone cómo evolucionan los sentimientos en la novela Orgullo y Prejuicio.

22 Como la extensión de los corpus analizados influye en la obtención del porcentaje de riqueza léxica, se han equiparado su longitud a 4560 palabras, que es la extensión del corpus más breve.

23 La capacidad de León Marchante para ganarse el beneplácito del público hizo que compusiera versos sacros para las Capillas Reales (1661-1679) y que se imprimieran múltiples pliegos sueltos de sus poesías religiosas (León, 2016). 
religiosas del maestro León. El porcentaje de emociones positivas que trasmiten estas últimas es muy similar al de los textos de autoría disputada.

Si se observa la riqueza léxica, las obras del obispo de Orense y las composiciones dubitadas albergan una mayor diversidad de vocabulario que las de Marchante ${ }^{24}$. Por último, se ha procedido a recopilar las «palabras distintivas ${ }^{25}$ que aparecen en los corpus de lírica indubitada (sacra y profana) y de prosa ${ }^{26}$ de ambos escritores para comprobar la presencia que estas tienen en cada uno de los poemas dubitados. Los resultados obtenidos se han recogido en dos tablas ${ }^{27}$ :

\section{TABLA 3. Palabras distintivas (I)}

\begin{tabular}{|c|c|c|c|c|c|c|c|}
\hline D_s2 & $D \_s^{3}$ & $\overline{D \_s 5}$ & D_s6 & D_s8 & D_s10 & D_s13 & D_s16 \\
\hline \multicolumn{8}{|c|}{ Palabras distintivas de Marchante } \\
\hline Encomiendas & Amarillo & Risa & Lado & Médico & Egipto & Arca & Arca \\
\hline \multirow[t]{6}{*}{ Montañes } & Cadena & Zarza & & & Gitano & Cascarón & Caña \\
\hline & Desierto & & & & Inclusa & Casta & Gritos \\
\hline & Danzar & & & & Labrador & Huevo & Lado \\
\hline & Jaque & & & & Tostado & Portátil & Llano \\
\hline & Preso & & & & & & Sabio \\
\hline & Recoleto & & & & & & Toros \\
\hline \multicolumn{8}{|c|}{ Palabras distintivas de Cornejo } \\
\hline Desnudez & Entero & Luzbel & Costado & Bulto & Marzo & Contenta & Ejecutar \\
\hline Oblea & Momento & Profundo & Señales & Postrada & Olla & Copete & Guardián \\
\hline Puño & Regalo & Torpeza & & & Pañal & Flaca & Guardado \\
\hline Querellas & Reverencia & & & & Saludador & Grana & Huraño \\
\hline Túnica & Tragedia & & & & Soledad & Milagros & Mañas \\
\hline \multirow[t]{5}{*}{ Yermo } & Travieso & & & & Tierno & Pobrete & Mil \\
\hline & Quizá & & & & & Profundo & Testigos \\
\hline & Vellón & & & & & Recogida & \\
\hline & & & & & & Vedar & \\
\hline & & & & & & Vale & \\
\hline
\end{tabular}

24 Todas las diferencias observadas sobre el léxico y la semántica en las poesías sacras de fray Damián y de Marchante coinciden con las obtenidas en la lírica profana indubitada e ambos autores.

${ }^{25}$ Se han considerado palabras distintivas aquellas que no comparten los corpus analizados (prosa y verso) de Cornejo y de Marchante. Se ha limitado la cantidad de estas a diez para la confección de las tablas con el propósito de no sobrepasar la extensión del artículo.

${ }_{26}$ Se ha constituido un corpus de prosa (treinta mil palabras) a partir de algunas de las cartas amorosas que el maestro León habría escrito, en un registro informal, a su prima monja (Foulché-Delbosc, 1903) y de diversos capítulos del tercer tomo de la Crónica seráfica (Cornejo, 1686).

27 Las palabras marcadas en cursiva se hallan en más de un poema dubitado. 
En casi todas las poesías que tratan hagiografías o eventos religiosos, se aprecia una mayor presencia de término distintivos de Cornejo. No obstante, en algunas como D_s3 o D_s10 el número de palabras que solo aparecen en los corpus de Marchante es bastante similar y en el D_si16 es igual. Sucede lo contrario con D_s2 y D_s13. En esta última composición, dedicada a santa Clara, resulta especialmente relevante la cantidad de léxico distintivo del cronista seráfico que posee, pues tal hecho (junto a los datos aportados en el análisis gramatical) ayudaría a refutar la teoría de Antonio Carreira que defiende que el maestro León podría ser el autor de esta ${ }^{28}$.

Respecto a la semántica de las palabras extraídas, cabe señalar que pueden observarse en ellas algunos patrones. Marchante parece mostrar una tendencia a incluir profesiones (médico, labrador o recoleto) y atributos o estados de individuos (sabio, preso y montañés), mientras que Cornejo tiende a describir rasgos relacionados con la psicología (travieso, tierno, huraño, pobrete o contenta) o con la apariencia externa (yermo, entero, profundo o desnudez). Tales características y la preeminencia de vocabulario distintivo de fray Damián se mantienen en el resto de textos analizados:

TABLA 4. Palabras distintivas (II)

\begin{tabular}{|c|c|c|c|c|c|c|c|}
\hline D_s7 & D_s9 & D_s12 & D_s14 & D_s17 & D_s18 & D_s22 & D_s23 \\
\hline \multicolumn{8}{|c|}{ Palabras distintivas de Marchante } \\
\hline & Limpieza & Cuerno & Festejar & Borrones & & Seglar & Alivio \\
\hline & Vicario & Luna & Iracundo & Guerra & & & Barro \\
\hline & & & & Limpieza & & & Lucimiento \\
\hline & & & & & & & Omisión \\
\hline & & & & & & & Trofeos \\
\hline & & & & & & & Veneno \\
\hline \multicolumn{8}{|c|}{ Palabras distintivas de Cornejo } \\
\hline Cerrado & Bola & Gloriosa & Ánimo & Admiración & Arrojar & Garra & Apetito \\
\hline Oscurecer & Cordel & Manzana & Codicia & Culebra & Cordel & Grano & Conocimiento \\
\hline Pensil & Extraordinario & Original & Dragón & Dragón & Codicia & Ponderación & Desfigurado \\
\hline Sello & Muerta & Querella & Fiereza & Luzbel & Pueblo & Postrada & Inútil \\
\hline \multirow[t]{5}{*}{ Serpiente } & Particular & Sano & Hermosura & Materia & & Reverencia & Misericordia \\
\hline & Pureza & Soberano & Sierpe & Sierpe & & Ruin & Mísero \\
\hline & Virginal & Trueca & Torpeza & Serpiente & & Siquiera & Obstinado \\
\hline & & & & Quebradero & & Tajada & \\
\hline & & & & & & Ufano & \\
\hline
\end{tabular}

28 Carreira (2008: 47-48) sostiene que en las Obras póstumas de Marchante (1722 y 1733) hay varios poemas indubitados dedicados a santa Clara en los que se pone en entredicho su castidad con juegos de palabras similares a los empleados en D_s13. 
En las poesías dedicadas a la Inmaculada Concepción (D_s7, D_s9, D_s12, D_s14 y D_s17) y en D_s18 y D_s22, la presencia de palabras distintivas de fray Damián es elevada y las del dramaturgo manchego casi nula. No ocurre lo mismo con D_s23, aunque en este poema sigue siendo superior el número de términos que Cornejo no comparte con Marchante. Es importante señalar también que las composiciones que defienden la pureza de la Virgen reiteran léxico distintivo del cronista seráfico: codicia, cordel, dragón, materia, serpiente y sierpe. Este hecho refuerza todos los datos, previamente comentados, que indican que pudieron ser escritas por fray Damián.

\section{ANÁlisis métrico}

Con el propósito de completar la caracterización de los poemas religiosos indubitados y dubitados de Marchante y de Cornejo, se ha procedido a delimitar las formas métricas que se utilizan en ellos. A pesar de que el número de textos seleccionados es bajo, conocer los porcentajes de estas puede aportar datos relevantes sobre sus preferencias en el uso de los metros y sobre si existen semejanzas respecto a su empleo en las poesías disputadas entre ambos. Los resultados obtenidos se han representado a través del siguiente gráfico:

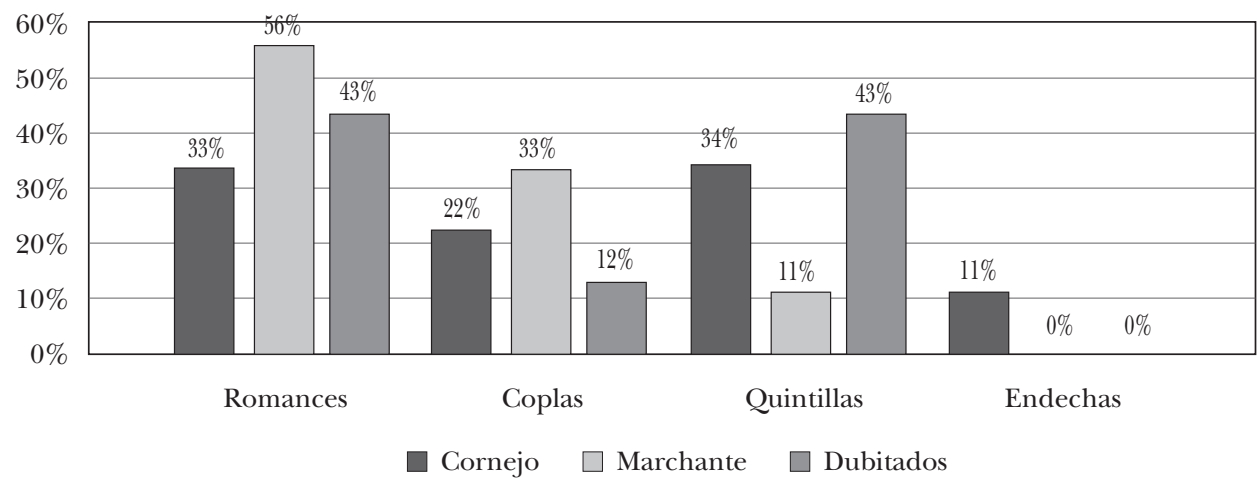

Gráfico 3. Análisis métrico.

Aunque en los tres corpus analizados predominan las quintillas, los romances y las coplas, se observan diferencias significativas en sus porcentajes. La proporción de quintillas en la poesía sacra indubitada de Cornejo ${ }^{29}$ triplica

${ }_{29}$ En la poesía profana indubitada de fray Damián los metros más empleados son los romances $(30 \%)$, los sonetos $(28 \%)$ y las décimas $(19 \%)$. Además, en las composiciones de temática humana se utiliza una mayor diversidad de formas métricas (ovillejos, silva o septeto), las cuales no adquieren un papel significativo en el total $(8 \%)$. 
la de Marchante, siendo este el metro más utilizado por el cronista seráfico. El dramaturgo manchego, por el contrario, realiza un mayor empleo de los romances $(56 \%)$ y de las coplas $(33 \%)$ y no usa endechas. Si se comparan dichos datos con los extraídos en las poesías religiosas disputadas entre ambos, los porcentajes de romances, quintillas y coplas son más similares a los de las poesías indubitadas de fray Damián ${ }^{30}$.

\section{Conclusiones}

Los avances que se están llevando a término en el procesamiento de lenguaje natural y en el análisis masivo de palabras ${ }^{31}$ permiten desarrollar estudios de corpus textuales más complejos y fiables. Estos ayudan a distinguir, mediante datos objetivos, incluso creaciones breves de escritores con un una formación y un contenido similar. Los estudios cuantitativos aplicados a la morfosintaxis, al léxico y a la métrica de las producciones literarias del Maestro León y de fray Damián han permitido singularizar sus poemas sacros y comparar sus rasgos estilísticos con las poesías religiosas disputadas entre ambos.

Gracias a los análisis de multivariantes, se ha podido visualizar las semejanzas en el uso de la gramática que existen entre las poesías divinas atribuidas exclusivamente a fray Damián y las dubitadas con el dramaturgo manchego. Además, estos han permitido obtener las medias de las principales categorías morfosintácticas que en ellas aparecen e identificar las que permiten diferenciarlas de las composiciones del maestro León: artículos, preposiciones, clíticos y posesivos. También se han observado similitudes importantes en el empleo de los bigramas entre los versos religiosos indubitados de Cornejo y los dubitados con Marchante, pues en ambos se aprecia un mayor interés por utilizar las combinaciones de un nombre, un adjetivo o un verbo con otro verbo.

El análisis cuantitativo del léxico y de la semántica ha ofrecido más datos relevantes sobre las características de los textos estudiados. Por un lado, ha puesto en evidencia que tanto los poemas divinos (dubitados e indubitados) de fray Damián contienen una mayor riqueza léxica y más referencias a la primera persona que los del dramaturgo manchego. Por otro, ha permitido

${ }^{30}$ Los romances, las quintillas, los villancicos y las seguidillas son las formas métricas más recurrentes en la lírica religiosa cómico-festiva del Bajo Barroco español. Exceptuando las quintillas, el resto de estas están «históricamente ligadas en sus orígenes a la producción lírica popular y tradicional, de difusión básicamente oral y mayoritariamente escrita para ser cantada» (López, 2011: 77).

31 El procesamiento masivo de corpus textuales text mining abre el camino hacia el análisis distante (Moretti, 2013) y el macroanálisis (Jockers, 2013) de las obras literarias. 
mostrar que las poesías sacras indubitadas de este último poseen una mayor voluntad de conectar con el receptor y que retratan más sentimientos negativos que el resto de corpus analizados. Por último, el análisis de las palabras distintivas ha puesto de manifiesto que comparten un mayor número de estas los poemas sacros disputados entre ambos con las obras en prosa y en verso (profano y sacro) del cronista seráfico.

Tras valorar todos los datos gramaticales, léxicos y métricos obtenidos, se puede concluir que las poesías dubitadas que se han analizado poseen más semejanzas estilísticas con las obras escritas por Cornejo. No obstante, la similitud temática con las producciones divinas de Marchante y el hecho de que pudieron difundirse entre los mismos círculos propiciaron que se acabaran publicando poco después de sus muertes con el nombre de este último, que era un poeta consagrado. Tal suceso no sería un caso aislado, ya que, como afirma Antonio Carreira (2001: 45), en el periodo aurisecular se producía una especie de gravitación con las composiciones líricas disputadas entre dos autores «el ingenio de mayor renombre en su tiempo es quien las atrae. Por ello, la norma filológica ha llegado a la conclusión de que cuando un poema se atribuye a dos ingenios, uno famoso y otro desconocido, este será el autor más probable».

Como se ha intentado demostrar a lo largo de esta investigación, los análisis cuantitativos aplicados a la gramática y al léxico pueden ayudar no solo a la determinar la verdadera autoría de las poesías dubitadas (sacras y profanas) que existen entre el maestro León y fray Damián, también pueden servir para resolver problemas de autoría de otros escritores. Si se desea seguir avanzando en este tipo de estudios, es importante que continúen desarrollándose herramientas y formas de análisis que permitan extraer información objetiva sobre el contenido y las características estilísticas de corpus textuales.

\section{BibLIOGRAFÍA}

Bègue, A. (2010): «Relación de la poesía española publicada entre 1648 y 1750». En Egido, A. y Laplana, J.E. (eds.): La luz de la razón: literatura y cultura del siglo XVIII: a la memoria de Ernest Lluch. Zaragoza, Institución «Fernando el Católico», págs. 399-476.

Burrows, J. y Love, H. (1999): «Attribution Tests and the Editing of SeventeenthCentury Poetry». The Yearbook of English Studies, Cambridge, Modern Humanities Research Association, 29, págs. 151-175.

Carreira, A. (2001): «El manuscrito como transmisor de las Humanidades en los Siglos de Oro». Boletín del Instituto de Investigaciones Bibliográficas, 6.1-2, págs. 21-46.

- (2008): «La obra poética de Damián Cornejo: cuatro manuscritos más y uno menos». Criticón, 103-104, págs. 39-54. 
Cornejo, D. (1686): Crónica seráfica y vida del glorioso patriarca San Francisco y de sus primeros discípulos, 3. Madrid, Imprenta de Juan García Infanzón.

- (1978): Das lyrische Werk des Damian Cornejo (1629-1707). Erster Teil. P. Klaus (ed.). München, Wilhelm Fink.

Craig, H. (2012): «George Chapman, John Davies of Hereford, William Shakespeare, and "A Lover's Complaint"». Shakespeare Quarterly, 63.2, págs. 147-174.

Craig, H. y Kinney, A. (2009): Shakespeare, Computers, and the Mystery of Authorship. New York, Cambridge University Press.

Eder, M. (2015): «Does size matter? Authorship attribution, small samples, big problema». Literary and Linguistic Computing, 30.2, págs. 167-182.

— (2017): «Short Samples in Authorship Attribution: A New Approach». Digital Humanities (en línea: < https://dh2017.adho.org/abstracts/341/341.pdf> [consulta: 6 de marzo de 2019]).

Foulché-Delbosc, R. (1903): «Coplas de Trescientas cosas más». Revue Hispanique, 10, págs. 234-235.

Jockers, M. (2013): Macroanalysis. Digital Methods and Literary History. Illinois, University of Illinois Press.

León Marchante, M. (1662): Letras de los villancicos de Navidad que se han de cantar en la Santa Iglesia de Toledo. Toledo, Imprenta de Francisco Calvo.

- (1722): Obras poéticas póstumas que a diversos asuntos escribió el maestro León Marchante, 1. Madrid, Real Capilla de su Majestad.

- (2016): Manuel de León Marchante. Obras complutenses. M.V. Sánchez (ed.). Alcalá de Henares, Institución de Estudios Complutenses.

Mata Induráin, C. y Bègue, A. (2018): «Introducción». En Bègue, A. y Mata Induráin, C. (eds.): Hacia la Modernidad. La construcción de un nuevo orden teórico literario entre Barroco y Neoclasicismo (1651-1750). Vigo, Academia del Hispanismo, págs. 13-22.

Moretti, F. (2013): Distant Reading. London, Verso.

OpArA, K. (2015): «Grammatical rhymes in Polish poetry: A quantitative analysis». Digital Scholarship in the Humanities, 30, 4, págs. 589-598.

Rezaei, S. y Kashanian, N. (2017): «A stylometric analysis of iranian poets». Theory and Practice in Language Studies, 7.1, págs. 55-64.

SÁnchez, Z. (2018): «Análisis cuantitativos aplicados a la poesía: la lírica indubitada sacra de Damián Cornejo y de León Marchante». Studia Aurea, 12, págs. 287-306.

Silgen, J. (2016): «You must allow me to tell you how ardently I admire and love Natural Language Processing» (en línea: <https://juliasilge.com/blog/you-mustallow-me> [consulta: 6 de marzo de 2019]).

Smarandache, F. (2007): «Linguistic-Mathematical Statistics in Recent Romanian Poetry». En Smarandache, F.: Collected Papers 1. Ann Arbor, InfoLearnQuest, págs. 184-191. 


\section{Anexo}

Tabla 5. Corpus de poesía sacra dubitada

\begin{tabular}{|l|l|c|l|l|}
\hline \multicolumn{5}{|c|}{ Poesía dubitada sacra } \\
\hline \multicolumn{1}{|c|}{ Primer verso } & \multicolumn{1}{c|}{ Lolización } & Extensión & \multicolumn{1}{c|}{ Métrica } & Nombre \\
\hline ¡Ah, Señor, el de la hostia! & BNE: Mss/ 2245 (fol. 183v) & 285 & Copla & D_s2 \\
\hline Al señor Bautista el jaque & BNE: Mss/ 2245 (fol. 169v) & 517 & Romance & D_s3 \\
\hline De Francisco por menor & BNE: Mss/ 2245 (fol. 165v) & 271 & Quintilla & D_s5 \\
\hline De pie quebrado unas coplas & BNE: Mss/ 2245 (fol. 164) & 202 & Copla & D_s6 \\
\hline De tu Concepción, Señora & BNE: Mss/ 2245 (fol. 158) & 214 & Romance & D_s7 \\
\hline Después que porque se usa & BNE: Mss/ 2245 (fol. 84v) & 156 & Romance & D_s8 \\
\hline De una niña la pureza & BNE: Mss/ 2245 (fol. 161) & 291 & Romance & D_s9 \\
\hline Escriben desde Toledo & BNE: Mss/ 2245 (fol. 177) & 308 & Romance & D_s10 \\
\hline Hanme mandado una cosa & BNE: Mss/ 2245 (fol. 154) & 188 & Quintilla & D_s12 \\
\hline Hoy mi devoción aclama & BNE: Mss/ 2245 (fol. 167) & 342 & Quintilla & D_s13 \\
\hline La Concepción este día & BNE: Mss/ 2245 (fol. 157) & 188 & Quintilla & D_s14 \\
\hline Otra vez tomo la pluma & BNE: Mss/ 2245 (fol. 152) & 349 & Quintilla & D_s16 \\
\hline Oigan lo que saca en limpio & BNE: Mss/ 2245 (fol. 195v) & 340 & Romance & D_s17 \\
\hline Por tomar Judas el grado & BNE: Mss/ 2245 (fol. 107) & 235 & Quintilla & D_s18 \\
\hline Un devoto singular & BNE: Mss/ 2245 (fol. 149v) & 192 & Quintilla & D_s22 \\
\hline Un pecador soy, Dios mío & BNE: Mss/ 2245 (fol. 172) & 394 & Romance & D_s23 \\
\hline
\end{tabular}


Tabla 6. Corpus de poesía sacra indubitada

\begin{tabular}{|c|c|c|c|c|}
\hline \multicolumn{5}{|c|}{ Poesía indubitada sacra } \\
\hline Primer verso & Localización & Extensión & Métrica & Nombre \\
\hline \multicolumn{5}{|c|}{ Damián Cornejo } \\
\hline En tu alabanza mi musa & BNE: Mss/ 2245 (fol. 185r) & 164 & Romance & C_sil \\
\hline De santa resurrección & BNE: Mss/ 2245 (fol. 186r) & 378 & Romance & C_si2 \\
\hline En títulos, Virgen pura & BNE: Mss/ 2245 (fol. 198r) & 294 & Quintilla & C_si3 \\
\hline Dulce Jesús, Dios mío & BNE: Mss/ 2245 (fol. 190r) & 166 & Endecha & C_si4 \\
\hline Hoy con gusto a cantar llego & BNE: Mss/ 2245 (fol. 200r) & 166 & Quintilla & C_si5 \\
\hline Oigan, que el bravo del cielo & BNE: Mss/ 2245 (fol. 191v) & 189 & Romance & C_si6 \\
\hline Divina Madre de Gracia, & BNE: Mss/ 2245 (fol. 188v) & 382 & Romance & C_si7 \\
\hline Haced que la Gracia encuentre & BNE: Mss/ 2245 (fol. 196r) & 116 & Quintilla & C_si8 \\
\hline A su esposo, el alma, escribe & BNE: Mss/ 245 (fol. 193v) & 191 & Copla & C_si9 \\
\hline \multicolumn{5}{|c|}{ León Marchante } \\
\hline En la noche más helada & León (1722: 17) & 244 & Romance & L_sil \\
\hline Atención, que hoy de José & León (1722: 46) & 372 & Romance & L_si2 \\
\hline En la noche más oscura, & León (1662: s.p.) & 175 & Copla & L_si3 \\
\hline ¡Oh qué bien que navega & León (1662: s.p.) & 298 & Copla & L_si4 \\
\hline Hoy un Francisco hace fiesta & León (1722: 38) & 243 & Romance & L_si5 \\
\hline Pendiente a morir, de un leño & León (1722: 26) & 194 & Romance & L_si6 \\
\hline La vida quiero contar & León (1722: 43) & 225 & Quintilla & L_si7 \\
\hline Vaya de jácara, y sea & León (1722: 9) & 242 & Romance & L_si8 \\
\hline Maravillas del Señor & León (1722: 12) & 239 & Copla & L_si9 \\
\hline
\end{tabular}

En las siguientes tablas se recogen los resultados de los análisis de multivariantes realizados a partir de los porcentajes morfosintácticos. En el análisis de clasificación clúster las composiciones de Marchante conforman el «Grupo 1», mientras que todos los poemas dubitados y los indubitados de Cornejo constituyen el «Grupo 2». Lo mismo sucede en el de discriminantes. Tales datos manifiestan las similitudes y diferencias en el uso de la gramática que existen entre las poesías analizadas. 
Tabla 7. Análisis de clasificación y de discriminantes de los poemas dubitados e indubitados sacros de fray Damián y del maestro León

\begin{tabular}{|l|c|c|}
\hline \multicolumn{3}{|c|}{ Análisis de clasificación } \\
\hline Case Number & Cluser & Distance \\
\hline D_s2 & 2 & 8,850 \\
\hline D_s3 & 2 & 3,097 \\
\hline D_s5 & 2 & 3,023 \\
\hline D_s6 & 2 & 7,156 \\
\hline D_s7 & 2 & 3,327 \\
\hline D_s8 & 2 & 11,227 \\
\hline D_s9 & 2 & 5,556 \\
\hline D_s10 & 2 & 7,868 \\
\hline D_s12 & 2 & 5,332 \\
\hline D_s13 & 2 & 4,197 \\
\hline D_s14 & 2 & 5,929 \\
\hline D_s16 & 2 & 4,579 \\
\hline D_s17 & 2 & 5,040 \\
\hline D_s18 & 2 & 4,810 \\
\hline D_s22 & 2 & 6,972 \\
\hline D_s23 & 2 & 7,925 \\
\hline D_s24 & 2 & 6,640 \\
\hline D_s25 & 2 & 9,764 \\
\hline D_s26 & 2 & 7,016 \\
\hline D_s27 & 2 & 9,616 \\
\hline C_si1 & 2 & 9,839 \\
\hline C_si2 & 2 & 8,091 \\
\hline C_si3 & 2 & 6,065 \\
\hline C_si4 & 2 & 5,776 \\
\hline C_si5 & 2 & 9,144 \\
\hline C_si6 & 2 & 6,237 \\
\hline C_si7 & 2 & 7,005 \\
\hline C_si8 & 2 & 7,439 \\
\hline C_si9 & 2 & 5,770 \\
\hline L_si1 & 1 & 4,922 \\
\hline L_si2 & 1 & 5,457 \\
\hline L_si3 & 1 & 6,318 \\
\hline L_si4 & 1 & 8,688 \\
\hline L_si5 & 1 & 5,696 \\
\hline L_si6 & 1 & 6,718 \\
\hline L_si7 & 1 & 6,403 \\
\hline L_si8 & 1 & 3,386 \\
\hline L_si9 & 1 & 11,495 \\
\hline & & \\
\hline
\end{tabular}

\begin{tabular}{|c|c|c|c|}
\hline \multicolumn{4}{|c|}{ Análisis de discriminantes } \\
\hline Case & Number & Actual Group & Predicted Group \\
\hline \multirow[t]{38}{*}{ Original } & D_s2 & 2 & 2 \\
\hline & D_s3 & 2 & 2 \\
\hline & D_s5 & 2 & 2 \\
\hline & D_s6 & 2 & 2 \\
\hline & D_s7 & 2 & 2 \\
\hline & D_s8 & 2 & 2 \\
\hline & D_s9 & 2 & 2 \\
\hline & D_s10 & 2 & 2 \\
\hline & D_s12 & 2 & 2 \\
\hline & D_s13 & 2 & 2 \\
\hline & D_s14 & 2 & 2 \\
\hline & D_s16 & 2 & 2 \\
\hline & D_s17 & 2 & 2 \\
\hline & D_s18 & 2 & 2 \\
\hline & D_s22 & 2 & 2 \\
\hline & D_s23 & 2 & 2 \\
\hline & D_s24 & 2 & 2 \\
\hline & D_s25 & 2 & 2 \\
\hline & D_s26 & 2 & 2 \\
\hline & D_s27 & 2 & 2 \\
\hline & C_sil & 2 & 2 \\
\hline & C_si2 & 2 & 2 \\
\hline & C_si3 & 2 & 2 \\
\hline & C_si4 & 2 & 2 \\
\hline & C_si5 & 2 & 2 \\
\hline & C_si6 & 2 & 2 \\
\hline & C_si7 & 2 & 2 \\
\hline & C_si8 & 2 & 2 \\
\hline & C_si9 & 2 & 2 \\
\hline & L_sil & 1 & 1 \\
\hline & L_si2 & 1 & 1 \\
\hline & L_si3 & 1 & 1 \\
\hline & L_si4 & 1 & 1 \\
\hline & L_si5 & 1 & 1 \\
\hline & L_si6 & 1 & 1 \\
\hline & L_si7 & 1 & 1 \\
\hline & L_si8 & 1 & 1 \\
\hline & L_si9 & 1 & 1 \\
\hline
\end{tabular}

Atribución: 1 León Marchante 2 Damián Cornejo 
En el siguiente biplot, se muestra cómo las poesías sacras indubitadas de Cornejo y las de Marchante presentan características morfosintácticas que permite diferenciarlas. En los cuadrantes superiores, se sitúan las producciones del dramaturgo manchego y, en los inferiores, se hallan los poemas de fray Damián:

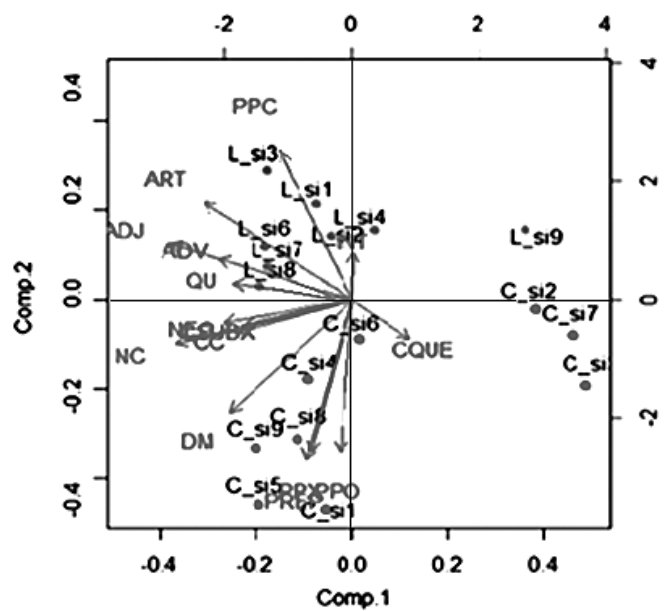

Gráfico 4. Análisis morfosintáctico de la poesía sacra indubitada de Cornejo y Marchante. 